\title{
Pascal Quignard : tours et détours d'une haine
}

\section{Pauline Vachaud}

\section{OpenEdition}

Journals

Édition électronique

URL : http://journals.openedition.org/recherchestravaux/444

DOI : 10.4000/recherchestravaux.444

ISSN : 1969-6434

\section{Éditeur}

UGA Éditions/Université Grenoble Alpes

\section{Édition imprimée}

Date de publication : 15 mai 2011

Pagination : 37-52

ISBN : 978-2-84310-200-4

ISSN : 0151-1874

\section{Référence électronique}

Pauline Vachaud, «Pascal Quignard : tours et détours d'une haine », Recherches \& Travaux [En ligne] 78 | 2011, mis en ligne le 15 novembre 2012, consulté le 08 septembre 2020. URL : http://

journals.openedition.org/recherchestravaux/444 ; DOI : https://doi.org/10.4000/recherchestravaux 444

(C) Recherches \& Travaux 
Pauline VACHAUd

Université Grenoble 3

\section{Pascal Quignard : tours et détours d'une haine}

Dans le IX traité de La Haine de la musique, intitulé «Désenchanter », alors que l'ensemble de l'ouvrage se tient plus ou moins fermement à la tension posée entre haine et musique, le fil des fragments dévie franchement, pour évoquer la place et le rôle de la critique d'art, et, plus particulièrement, de la critique littéraire :

Les œuvres font peur aux codes. La haute mer fait peur aux mouettes comme les rats préferent les égouts des villes populeuses.

Ceux qui émettent un jugement demeurent toujours sur le rivage.

En criant, ils suscitent les naufrages qu'ils appellent de leurs vœux.

C'est le cri aigre des oiseaux de mer qui survolent l'écume blanche des vagues noires de la mer. Ce cri est plein de détresse. Ils cherchent des détritus pour manger. Ils cherchent des épaves où se poser. [...]

Les gardiens du conditionnement moral, esthétique, politique, religieux, social ont toujours raison; ils veillent au contrôle symbolique du groupe.

Anna Akhmotova appelait les critiques dans les journaux et les professeurs de lettres dans les écoles les "gardiens de prison». (HM, p. 27I-273.)

On sait que chez Pascal Quignard les motifs de la mer, du naufrage, le thème de la mort et la question de l'aliénation sociale sont récurrents, quel que soit l'objet de la réflexion, mais ce qui est surprenant ici, c'est le déplacement du propos auquel ils contribuent : de la haine de la musique qui oriente les dix traités du livre, une digression nette est opérée, soulignant l'écueil de la critique - la haine qui la fonde et qu'elle suscite en retour.

Sous de tels auspices, proposer une analyse des fluctuations de l'imaginaire musical, et, plus particulièrement, des traits de la haine dans La Haine

I. La Haine de la musique [1996], Gallimard, coll. «Folio», 2002. (Désormais HM.) 
de la musique et Boutès ${ }^{2}$ peut être de l'ordre du défi. Je voudrais cependant le relever, en essayant de ne pas étouffer la "pensée vivante» ( $B$, p. 2I) qui traverse l'écriture de l'auteur. Dans un premier temps, il s'agira d'évoquer les conditions formelles de cet imaginaire fluctuant, autrement dit, ce que nous pourrions appeler la dimension analytique de l'écriture - ce en quoi l'écriture, chez Pascal Quignard, procède par glissements, associations libres, retournements et paradoxes, en étant régie par une logique autre, plus proche de l'inconscient ou, du moins, de la mythologie personnelle que de la raison rationnelle, objective ${ }^{3}$. Ensuite, j'appréhenderai plus précisément les différentes figures de cet imaginaire musical - figures si variées et contradictoires qu'au fond, semble-t-il, quiconque peut y trouver de quoi nourrir ses propres positions. Pour autant, malgré les variations et retournements au gré desquels se déroule la conception musicale dans ces deux ouvrages, et malgré la réconciliation apparente que semble signer Boutès après la haine du premier, un point de convergence fondamentale réunit ces deux textes : de l'un à l'autre, Quignard n'offre finalement à son lecteur guère d'issue à l'aimantation musicale - ce terreau de l'amour comme de la haine.

\section{Dérives de l'écriture}

Les traités de Pascal Quignard, on le sait, ne relèvent pas d'un discours qui respecterait les règles de la démonstration rationnelle, méthodique, progressive, orientée autour d'une thèse bien précise. Ce qui est à l'œuvre dans cette esthétique qui témoigne plus de l'art du trait que de l'élaboration réflexive cadrée, c'est une logique de l'insistance, de l'obsession, où la pensée, la logique et la vérité sont assumées comme matière vivante - évolutive, malléable poreuse - et subjective. En cela l'écriture suit des chemins volontiers tortueux, déterminés par le glissement et le retournement. De ces aspects stylistiques sur lesquels reposent les «spéculations» propres à Quignard, nous ne retiendrons que quelques exemples.

Dans La Haine de la musique, tout d'abord, on peut discerner trois types de glissements : à l'échelle lexicale, propositionnelle et temporelle.

2. Boutès, Galilée, coll. «Lignes fictives", 2008. [Désormais B.]

3. En cela, le sens que je donne ici à "analytique» diffère de celui déplié par Boutès (p. 15), où l'analyse (la psychanalyse) est pour une fois mise du côté de l'ordre social et d'une certaine logique civilisatrice. L'arrière-plan psychanalytique était néanmoins une dimension indéniable du travail de Pascal Quignard, je conserverai cette référence, avec la force subversive (de la pensée, du sujet, du langage) qu'elle engage. 
Les jeux de paronomase, très souvent redoublés par une pratique de la dérivation, sont en effet une des pierres de touche de l'écriture quignardienne. Ce cheminement du sens selon la proximité sonore des mots, un des fragments du I ${ }^{\text {er }}$ traité, "Les larmes de saint Pierre», le manifeste clairement. Le couple "Tréô et terrere» sur lequel s'ouvre la page (HM, p. 4I) appelle immédiatement, en résonance, "Trémô et tremere». Et tout le fragment de dérouler divers types de tremblement, jusqu'à celui des œufs mollets, les «tremula ova». Si un certain sens relie ces différentes acceptions, malgré l'incongruité apparente de leur rapprochement, ce nœud de signification se fonde d'abord sur un accolement sonore ("tréô»/«trémô», et "terrere»/«tremere»). Un peu plus loin, ces effets de dérive signifiante s'accompagnent d'un tour supplémentaire : quand le chant du coq du reniement de saint Pierre est désigné comme un "pavé" de Venise, un expavanté au sein de l'expérience sonore du langage» ( $H M$, p. 89), c'est la création d'un néologisme, où résonnent le "pavé» et la "pavor», qui accentue ce glissement du sens sous le son.

Par ailleurs, le goût pour l'association libre ne touche pas que l'échelle du mot. Le lien entre les propositions procède volontiers par un fil analogique plus que par celui de la logique pure. À cet égard, le fragment qui assimile le sourire, la panique et la musique est particulièrement radical :

L'aimant fait venir à lui spontanément les minuscules débris de fer, de cobalt ou de chrome. L'aimant est comme le sourire de la mère. Le sourire de la mère entraîne sur-le-champ l'imitation d'un retroussis de lèvres sur la face de l'enfant. Le sourire de la mère est comme la peur : dans la peur la contagion s'appelle panique. Nous sommes tous, dès le surgissement, avant le surgissement, dès la plus extrême enfance, avant la naissance elle-même totalement mimétiques, aussi reproducteurs que nos mères l'ont été pour nous faire. Nous sommes tous totalement paniqués. La musique est comme le sourire panique. Toute vibration qui approche le battement du cœur et le rythme du souffle entraîne une même contraction, aussi involontaire, aussi irrésistible, aussi panique. [...] Nous sommes tous sans résistance à l'égard de la panique (la pierre panique, le sourire de la mère magnétique, le pôle panique, la boussole mentale. [...]) (HM, p. 53-54.)

Ici, la préférence pour l'analogie n'est pas seulement notable dans le martèlement des formules comparatives ("comme», "même», "aussi») et globalisantes («nous sommes tous [...]»). Elle tient aussi à la mise à plat des rapports entre thème et prédicat. Le thème posé à l'ouverture du fragment, "l'aimant", s'avère en fait, malgré le tour de la comparaison initiale ("L'aimant est comme le sourire de la mère.»), un ressort de la prédication pour un autre thème, le «sourire panique». Puis, selon cet "esprit d'escalier» (HM, p. 92) que Pascal Quignard reconnait lui-même à sa pensée, ce «sourire panique» devient à son tour le prédicat de la musique («La musique est comme le sourire panique.») Ce processus d'analogie selon lequel les pôles 
du thème et du prédicat se renversent et s'entremêlent, l'énumération finale des métaphores en rend compte au plus haut point ( la pierre panique, le sourire de la mère magnétique, le pôle panique, la boussole mentale»).

Enfin, à l'échelle temporelle, le recoupement de scènes historiques a priori distinctes, leur mise en concordance au-delà de la vraisemblance des situations, constitue un autre lieu essentiel à cette "espèce d'activité mythique» dans laquelle Pascal Quignard assume de vouloir de «tout replong[er]» (HM, p. 275). Ainsi, à partir de l'élément commun à la scène du reniement de saint Pierre et, chez Pétrone, au banquet de Trimalchio - le chant du coq - Pascal Quignard propose non pas seulement de relever la symétrie des deux moments, mais, plus radicalement, leur fusion :

Ces deux scènes romanesques associant l'action au chant d'un coq de basse-cour forment d'étranges miroirs. [C]ette symétrie est d'autant plus fascinante qu'une imagination un peu érudite, transportant de façon malaisée ce banquet sous le règne de Tibère, pourrait s’ingénier à la fonder historiquement. On peut conjecturer que c'est la même année. On peut avancer que c'est le même jour. On peut supposer que c'est la même heure. On peut peut-être dire que c'est le même coq. (HM, p. 94.)

Ailleurs, l'assimilation de saint Pierre à un homme paléolithique ${ }^{4}$ ou encore la figuration de cette scène du reniement dans un style baroque, sous Henri IV ou Louis XIIIs, entérine cette propension à l'écrasement des différences temporelles.

Dans Boutès, cet élan vers une écriture analogique, par glissement, est d'autant mieux mise en ouvre que tout l'ouvrage, au fond, thématise la tension entre la pensée qui délie - la pensée abstraite, articulée, logique, symbolisée par Ulysse - et la pensée qui relie, en jeu dans la musique et incarnée par Boutès :

Comment la musique pense-t-elle? Comme s'avance-t-elle dans la pensée? Pour nommer sans trop de prétention la pensée appelons-la la «reliaison». La pensée est ce qui relie les absents, les mots, les arguments, les impressions, les souvenirs, les images. Comme la reliaison suppose la liaison, la pensée suppose la mère. Pour nommer la mère disons la lieuse. Où on retrouve la seirèn. Vieille sirène qui erre au sein d'un vieux chant continu de base 2.» $(B$, p. 64.)

4. «Dans La Tour, le corps de saint Pierre est curieusement, non pas dressé, d'homme fait, voyant de haut la servante, la fuyant, mais de vieillard, accroupi, écrasé, à taille d'enfant, les genoux au menton, comme les morts que les hommes de l'époque paléolithique liaient avec des nerfs de renne, sous la forme ramassée des foetus, dans l'espoir d'une seconde naissance dans l'ombre de la peau de la bête totémique.» (HM, p. 96.)

5. «Les larmes de saint Pierre, quelque effort que je fasse pour mettre sous les yeux une scène plus romaine, je ne puis me la figurer que dans le style baroque, sous Henri IV ou sous Louis XIII. C'est une cour du Louvre l'hiver, grise, ou une cour pluvieuse dans Rouen. Ou une cour humide et glaciale dans le Lunéville de La Tour.» (HM, p. 96.) 
Il n'est alors pas anodin que soit d'emblée mise de côté la figure d'Ulysse, celui qui a "passé sans mourir le Lien des lieuses", les "Seiréniennes", et dont l'expérience représente la "première "analyse" [...], l'instant où sont dénoués les nœuds» ( $H M$, p. I5). Face à ce symbole de la déliaison ${ }^{6}$ qui fonde toute la culture occidentale, privilégier Boutès, c'est une manière d'indiquer sinon le ressort même de l'écriture, du moins la référence sous laquelle elle souhaite se placer.

Sans reprendre une à une les formes du glissement - de la reliaison - mises en œuvre par le texte, il suffit de porter l'attention sur l'évocation d'Orphée, au chapitre XIII, pour prendre la mesure de cette préférence chez l'auteur lui-même. Proposant un premier parallèle avec le mythe shintô d'Izanagi et Izanami à partir du motif de la descente aux enfers, Pascal Quignard poursuit son élan "symétrique» en soulignant la proximité frappante entre la mort d'Orphée et la mort de Boutès : "Je veux juste montrer que la mort d'Orphée est la scène symétrique de la mort de Boutès dans le chant IV d'Apollonios. » (B, p. 69.) Alors qu’Orphée, au début de l'ouvrage, est donné comme la figure inversée de Boutès, au moment de sa mort, quand ressurgissent la femme originaire (sous la forme des bacchantes) et le bain dans l'eau du jadis (la tête décapitée du musicien gisant dans l'Hèbre), les obsessions du texte font retour, consommant la reliaison avec l'histoire de Boutès.

D'autre part, à ces formes de glissements analogiques sur lesquelles repose la rhétorique de La Haine de la musique et de Boutès, il faut ajouter un goût assumé pour le retournement et le paradoxe. Dans La Haine de la musique, par exemple, la vertu de l'inversion est explicitement valorisée, à propos, notamment de la scène d'Ulysse et les sirènes : «le simple fait d'inverser l'épisode me paraît lui donner son sens le plus sûr. [D]es hommes attirent par un chant artificiel des oiseaux dans le lieu jonché d'os où ils nichent ${ }^{7}$ » (HM, p. 167). Quant à Boutès, il ne ménage certes pas un tel renversement du mythe, mais l'attention à ce personnage «ostracisé» qui symbolise le refoulé, l'impensé, l'insoumis relève de cette même démarche à contre-courant :

Il y a des oubliés au souvenir du monde. Il faut céder un peu d'eau pure, c'està-dire un peu de langue écrite, aux vieux noms qu'on ne prononce plus. [...] il faut laisser une chaise vide à ceux qui ont été injustement ostracisés. [...] Boutès

6. Dans cet ordre d'idées, à la fin de l'ouvrage, c'est comme "Contre-Sirène» et «Déliante" qu'est évoquée La Délie de Maurice Scève ( $B$, p. 83).

7. Voir aussi, dans le I $\mathrm{I}^{e}$ traité : «On dit de certaines pluies qu'elles martèlent. D’autres qu'elles tambourinent. D'autres qu'elles crépitent. Ces images, indépendamment de l'impression de vérité qu'elles procurent, sont à proprement parler extraordinaires - un tambourin, un feu, un marteau - pour dire la pluie. De telles images conduisent alors à inverser la comparaison à leur source. Ce ne fut pas la pluie qui tambourinait. Ce fut le tambourin qui appelait la pluie.» (HM, p. 72-73.) 
avait quitté la compagnie des autres Argonautes. [...] Il n'avait suivi ni l'exemple ni l'ordre d'Orphée. (HM, p. 29-30.)

On trouve aussi, dans La Haine de la musique, un bel exemple d'antithèse radicale dans le commentaire de la version du reniement par saint Jérôme :

I. La correction de Jérôme est tout à fait justifiée. Saint Marc a raté le premier chant qui prépare l'émotion. Un musicien, un romancier ne l'eussent pas omis, pour l'effet pathétique que ce premier appel parait héler dans l'espace du texte, sans qu'il soit entendu encore par le héros.

2. La correction de Jérôme n'est pas du tout justifiée. Il y a dans ce chant déjà répété, et qui n'apparait pour la première fois qu’à la seconde, qui produit une triple trahison, une profondeur qui émeut et que je n'explique pas d'une façon claire. (HM, p. 91-92.)

Poser une idée et son contraire sans trancher, c'est une manière particulièrement remarquable d'œuvrer dans le sens d'une logique autre. Dans la même veine, par la place faite à certaines mystiques, comme celles de Maître Eckhart (HM, p. 243-246) ou du marchand Vamalakirti (HM, p. 264-267), le sens du paradoxe est clairement convoqué :

«Écoute le désert du son.» (HM, p. 244.)

«Il n’y a pas de langage qui nous parle. Il n'y a pas de silence qui le taise ${ }^{8} . »(H M$, p. 265.)

Quant à la solution envisagée par Quignard pour sortir de la répétition compulsive du passé, elle relève presque du comble :

Le statu quo ante hante le temps social. [...] La servilité et l'identité, qui sont des liens, prévalent sur la liberté et l'aventure, qui sont moins des possessions que des ivresses. J'avais imaginé une ruse afin de renoncer à faire de l'empreinte un destin. La thèse que je défendais vaille que vaille consistait à penser qu'il était possible qu'en s'appuyant sur beaucoup plus ancien que l'Histoire on pût se soustraire un peu à la répétition compulsive de son passé. ( $B$, p. 6I-62.)

Pour autant, dans ce recours au trait paradoxal, il ne s'agit pas simplement de céder à l'obscurité sibylline, au flottement pur du sens, lequel rendrait au lecteur une entière liberté d'interprétation. Déconstruire les catégories de la logique en tant que telle, ce n'est pas renoncer à tout discours de vérité, puisqu'il est question, en fait, d'éclairer des fonds structuraux refoulés. Mettre en avant l'union des contraires, c'est d'abord et avant tout relever combien la raison, et chaque instance censément positive portent en elles-mêmes leur revers :

8. Ailleurs, c'est Pascal Quignard lui-même qui assume l'énonciation de paradoxes similaires : "Je fuis la musique infuyable» $(B$, p. 26I), ou encore : "Celui qui écrit est ce mystère : un locuteur qui écoute» $(B, \mathrm{p} . \mathrm{132})$. 
L'art n'est pas le contraire de la barbarie. La raison n'est pas la contradictoire de la violence. On ne saurait opposer l'arbitraire à l'État, la paix à la guerre, le sang versé à l'affût de la pensée, parce que l'arbitraire, la mort, la violence, le sang, la pensée ne sont pas libres d'une logique qui demeure une logique si elle passe la raison. (HM, p. 220.)

Plus profondément, encore, l'indistinction des places entre sujet et objet contribue à cet autre type de pensée qui déjoue les règles de l'univocité. La duplicité de la valeur du génitif, présent dès le titre même La Haine de la musique (à entendre comme la haine inhérente à la musique et le caractère haïssable de la musique) place d'emblée l'ensemble du texte sous le signe de cette réversibilité. Et par deux fois cette indistinction qui concernerait tout particulièrement l'expérience musicale est explicitement posée :

Il n'y a pas de sujet ni d'objet de l'audition. (HM, p. I08.)

$\mathrm{Ni}$ interne ni externe, nul ne peut distinguer clairement dans ce qui déploie la musique ce qui est subjectif et ce qui est objectif, ce qui appartient à l'audition et ce qui appartient à la production du son. ( $H M$, p. I2I.)

Enfin, de l'un à l'autre ouvrage, un retournement fondamental est opéré, qui touche la qualité principale reconnue à la musique. Toute La Haine de la musique se déploie finalement sur le trait "Ouïr, c'est obéir» (HM, p. Io8), qui, plus loin, est déplié de la manière suivante :

Le sonore ne délimitant rien a moins individualisé les oreilles qu'il ne les a vouées à l'agroupement. Cela s'appelle : tirer par l'oreille. Hymnes nationaux, fanfares municipales, cantiques religieux, chants familiaux, identifient les groupes, associent les natifs, assujettissent les sujets.

Les obéissants. (HM, p. I2I-I22.)

La musique est donc assimilée à la passivité qu'elle provoque, et à sa violence, en vertu du caractère illimité du son mais aussi de ses manifestations originaires : la chasse, la domestication, la détermination aliénante de la «sonate maternelle» (HM, p. Io9). Cette obéissance terrible est alors ce que le traité "La haine de la musique» développe le plus radicalement, en évoquant les raisons pour lesquelles «la musique est le seul, de tous les arts, qui ait collaboré à l'extermination des Juifs [...], le seul art qui ait pu s'arranger de l'organisation des camps, de la faim, du dénuement, du travail, de la douleur, de l'humiliation, et de la mort» (HM, p. 197) :

La musique viole le corps humain. Elle met debout. Les rythmes musicaux fascinent les rythmes corporels. À la rencontre de la musique l'oreille ne peut se fermer. La musique, étant un pouvoir, s'associe de ce fait à tout pouvoir. Elle est d'essence inégalitaire. Ouïe et obéissance sont liées. Un chef, des exécutants, des obéissants, telle est la structure que son exécution met en place. Partout où il y a un chef et des exécutants; il y a de la musique. Platon ne pensait jamais à distinguer dans ses récits philosophiques la discipline et la musique, la guerre et le 
musique, la hiérarchie sociale et la musique. Même les étoiles : ce sont les Sirènes selon Platon, astres sonores producteurs d'ordre et d'univers. Cadence et mesure. La marche est cadencée, les coups de matraque sont cadencés, les saluts sont cadencés. La première fonction, ou du moins la plus quotidienne des fonctions assignées à la musique des Lagerkapelle consista à rythmer le départ et le retour des Kommandos. (HM, p. 202-203.)

Viol, pouvoir, inégalité, guerre, discipline, hiérarchie: selon un fatalisme implacable, tels seraient les actions et les attributs essentiels de la musique.

Dans Boutès en revanche, une opposition est formulée entre la musique originaire - l'espace du sonore représenté par les sirènes - et la musique orphique - instrumentale, civilisée, ordonnée, retenue, masculine, qui se joue et s'écoute assis. Selon cette division, c'est la musique orphique qui aurait à voir avec l'ordre imposé et la violence - sa haine et ce qui la rend haïssable :

La pensée d'Apollonios est claire. À ses yeux il y a deux musiques. L'une de perdition (qu'il définit admirablement en disant qu'elle ôte le retour), l'autre orphique, salvifique, articulée, collective, qui est celle qui procure son unanimité et qui de ce fait assure la rapidité aux rames des rameurs. Exclusivement humaine, ordonnée, ordonnante, elle ordonne le retour. Apollonios écrit qu'Orphée [...] prend de force le chant qui sidère. [...] La cithare a violé le chant des vierges. En violant la sidération Orphée viole le féminin marqué par leur voix. Bref Orphée contraint avec une violence exclusivement virile le chant acritique. ( $B$, p. I7-I8.)

La musique grecque puis romaine puis chrétienne se fit de plus en plus orphique et conjuratoire. Elle devint extraordinairement instrumentale. La musique occidentale sacrifia la danse originaire qui appartient néanmoins au noyau archaïque. C'est d'abord le délaissement de la transe puis c'est le renoncement à quitter le rang des rameurs qui autorisèrent son écriture. Qui expliquent son exécution assise mais surtout son inexplicable et pour ainsi dire "onirique» inhibition musculaire - sa prodigieuse audition assise. ( $B$, p. 30-3r.)

De là, «l'animalité antérieure» $(B$, p. 28), la pulsion insoumise en jeu dans la musique originaire en ferait sa liberté, sa dissidence :

Et c'est encore Boutès, c'est encore l'Argonaute, c'est encore le dissident qui plonge. Sedeo, c'est être assis sur son banc.

Dis-sideo c'est se dés-asseoir.

Le dis-sident se désassocie du groupe qui ne cherche à accompagner et à domestiquer le solitaire qu’à partir de sa naissance. [...]

Boutès a peut-être raison.

Il faut peut-être tourner le dos à la musique orphique, occidentale, technologique, populaire.

Il faut peut-être laisser ce qui fait ramer sur le banc des rameurs.

Il faut peut-être s'éloigner de l'efficacité sonore excessive.

Il faut peut-être s'écarter du «bruit du plectre»". (B, p. 33-35.)

9. Notons que dans La Haine de la musique, la dissidence est aussi valorisée, mais en étant reliée à une tout autre disposition : "Logos insinue du deux dans du un. En 520 après Jésus- 
Se "dés-asseoir", sortir du rang, laisser la cithare pour revenir «au chant des vierges " et plonger dans le "noyau archaïque» sont alors autant de traits qui, après la haine, seraient à même de refonder l'amour. De l'obéissance à la dissidence, de la haine à l'amour, le renversement est sans appel, même si le prix à payer semble le renoncement à la musique savante, ce qui est assez surprenant quand l'on sait le goût de Pascal Quignard en la matière.

Du fait de cette écriture qui suit les méandres d'une pensée par analogies et retournements, où les catégories de la raison n'ont guère de pertinence, qui veut discerner la conception de la musique en jeu chez Pascal Quignard, dans ces deux textes du moins, est bon gré mal gré contraint à renoncer à une telle ambition, et doit se contenter de repérer les pôles contradictoires autour desquels s'organisent les fragments. Ainsi, là où se rejoignent ces deux ouvrages, finalement, c'est dans la nette ambivalence de l'imaginaire musical.

\section{Ambiguïtés de l'objet}

Dans La Haine de la musique et dans Boutès, on note une perpétuelle oscillation entre une conception de la musique comme lieu en parfaite continuité avec le fond originaire de l'humain - son fond pulsionnel, animal, archaïque, inconscient - et, au contraire, une conception qui insiste sur le caractère définitivement perdu de cette origine, où la musique n'est plus qu'une manière de rechercher vainement cette origine, de s'en défendre, de commémorer sa perte ou aider à s'en consoler. Quelques exemples significatifs suffisent à montrer combien le caractère non systématique de la pensée de Pascal Quignard permet de fait à tout lecteur de trouver son compte.

Dans Boutès, tout d'abord, nous pouvons avoir l'impression d'une plus grande stabilité puisque le texte n'évoque pas la musique en général, comme dans La Haine, mais qu'il opère une distinction entre la musique originaire (la «nuit sonore» vers laquelle replonge Boutès) et la musique orphique comme symbole de la domestication sociale - cette seconde se défendant des tentations de la première en maintenant l'ordre, la tenue, l'obéissance. Néanmoins, au sein de cette partition qui semble clarifier le propos, les définitions de chaque tendance ne sont pas si tranchées. Concernant la musique originaire, notamment, on peut relever de lourdes ambiguïtés. Tantôt le désir de Boutès de «s'approcher» de la musique est désigné comme impossible :

Christ, le philosophe grec Damaskios, à Athènes [...] écrivait que tout logos était fondateur d'une royauté de dissidence dans un univers continu.» (HM, p. 33.) 
Boutès : rejoindre la condition originaire, c'est mourir.

C'est ainsi que la musique est bien une «île» au milieu de l'océan; une île dont toute approche est impossible sauf à périr noyé. $(B, \mathrm{p} .72$.

L'origine serait résolument perdue, et c'est pourquoi la passion en jeu dans la musique serait la douleur, «une plongée dans le deuil de la $\operatorname{Perdue}^{\mathrm{ro}}$ " $(B$, p. 19). En cela, la musique serait ce "plongeon où l'irrattrapable sans cesse n'est jamais rattrapé» $(B$, p. 55$)$, et la force de cette formule bien scandée réside dans la boucle sans faille que dessine la tautologie. L'expérience musicale serait donc de l'ordre de la nostalgie : "La musique est nostalgie, après l'apprentissage des langues collectives, de l'état antérieur de la phonation, du souffle, de l'animation, de l'anima, de la psyché.» ( $B$, p. 85.)

Tantôt, au contraire, ce bain dans le jadis est envisagé comme possible :

La musique renvoie à un jadis qui sans respirer - ou plutôt respirant avec les oreilles, respirant avec l'ouie - entendait au fond de l'eau.

Jankélévitch a écrit : La musique nous enveloppe et c'est ainsi qu'elle nous pénètre car elle est vaste et infinie comme la mer. (B, p. 76 .)

Et certains musiciens comme certains écrivains parviendraient à accomplir cette immersion :

Rares, très rares les humains qui se jettent à l'eau pour rejoindre la voix de l'eau, la voix infiniment lointaine, la voix pas même voix, le chant pas encore articulé qui vient de la pénombre.

Quelques musiciens.

Quelques écrivains plus silencieux que d'autres dans des pages plus muettes encore. ( $B$, p. 75-76.)

Que l'approche de cet impossible, cette plongée dans le bain originaire soit cependant évoquée comme pratique silencieuse - toucher la musique originaire par le biais du mutisme - là n'est pas, une fois encore, le moindre des paradoxes.

Cette ambivalence - mal du retour ou retour consommé à l'origine - n'est pourtant pas sans raison : elle est due à la fluctuation de la notion d'origine elle-même, parfois évoquée comme l'état avant la naissance, le bain intrautérin, l'archaïsme le plus reculé, parfois au contraire représentée comme moment de la naissance, de l'humanisation - autrement dit la déchirure, la séparation. Ainsi balancée entre ces deux définitions du jadis, la conception de la musique, dans Boutès, hésite donc entre une sorte de rousseauisme libertaire et un romantisme mélancolique.

Io. Sur ce point, on peut relever la très belle page consacrée à Schubert $(B$, p. 24). 
Quant à La Haine de la musique, elle met en œuvre des fluctuations analogues, d'autant plus prégnantes qu'elles ne sont pas sous-jacentes à une dichotomie apparente, comme dans Boutès. La musique dans son substrat pulsionnel et archaïque est représentée, par exemple, sous la forme d'Athéna qui, jouant de la flûte, a «la bouche distendue, les joues gonflées, les yeux exorbités» et, plus loin, "la bouche occupée» (HM, p. I5-I6). La comparaison d'Aristote reprise à ce sujet ne laisse aucun doute quant à la connotation sexuelle de la formule :

Aristote dit dans la Politique que la muse a la bouche occupée et les mains occupées exactement comme une prostituée qui regonfle à l'aide de ses lèvres et de ses doigts la physis de son client afin de la dresser au bas de son ventre en sorte qu'il émette sa semence ${ }^{\text {II }}$ ( $(H M$, p. I4.)

De même, dans la formule lapidaire où joue, significativement, une allitération appuyée: "tout est concert du sang lié au son» (HM, p. 49), la part résolument corporelle, presque viscérale de l'expérience sonore est mise en avant. Et c'est bien comme résurrection d'un fond archaïque nommé la "curiosité sonore», autre nom de «l'alerte animale» (HM, p. 2I), qu'est convoquée l'écriture harmonique : «La caractéristique de l'harmonie est de ressusciter la curiosité sonore défunte dès le langage articulé et sémantique s'étend en nous.» (HM, p. 22.)

Ailleurs, néanmoins, la musique est envisagée comme détour, défense, consolation eu égard au "pathétique du lien d'enfance» (HM, p. 19), cette "souffrance sonore» (HM, p. I6). Elle est mise au même niveau que la Consolatio de Boèce (HM, p. I8), selon, encore une fois, un jeu d'écho sonore signifiant : "la muse "amuse" la douleur» (HM, p. I7). L'évocation de Haydn sur laquelle se poursuit le chapitre relaie aussi l'image du compositeur se défendant, par la composition, de ce "pathétique du lien d'enfance». Plus loin, il s'agit encore de distinguer nettement la musique du sonore, en relevant le rapport de maittrise, ou de protection, de l'une à l'autre :

Dans les instants les plus rares, on pourrait définir la musique : quelque chose de moins sonore que le sonore. Quelque chose qui lie le bruyant. (Pour le dire autrement : un bout de sonore ligoté. Un bout de sonore dont la nostalgie entend demeurer dans l'intelligible.) (HM, p. 24.)

Curieusement : la musique protège des sons. Les premières œuvres de la musique dite baroque étaient habitées par la volonté de s'extirper de l'aboi du sonore à

II. Il est significatif que le même motif soit repris dans Boutès : "[Alcibiade] dit à son mâ̂tre : "La flûte comme le chant sont des pratiques indignes. L'une et l'autre gonflent les joues et défigurent l'harmonie du visage humain. En revanche l'usage du plectre et de la lyre ne portent atteinte ni au maintien, ni au visage, ni à la liberté du mouvement d'un homme libre. [...] Quand on joue de la lyre on peut parler en même temps qu'on fait de la musique." " (B, p. 27-28.) 
partir de la modulation propre au langage humain et à l'organisation des affetti. L'invention de l'opéra résulta de cette volonté de renaissance affective, de mue ou de tri sonore, de sacrifice sonore. (HM, p. IOI-IO2.)

Cette ambivalence entre continuité et séparation, un paragraphe du «chant des sirènes" la condense subtilement :

La musique ne consiste nullement à faire entrer dans une ronde humaine; elle fait pénétrer dans une ronde zoologique reproduite. [...] Les sons mimés, qui sont les masques sonores des proies, font entrer l'animal céleste, l'animal terrestre, l'animal aquatique, tous les animaux prédateurs y compris l'homme, le tonnerre, le feu, le vent, dans la ronde prédatrice. La musique fait circuler la ronde par les sons des bêtes dans la danse [...] Elle en intensifie la rotation. (HM, p. 170-17I.)

Bien que la musique soit un "son mimé» - un leurre du sonore séparé de lui -, elle participerait pourtant à cette "ronde prédatrice», ce mouvement originaire du monde.

Par ailleurs, les attributs divins du sonore - son caractère «invisible», «immatériel», «illimitant», «inlocalisable», «indélimitable»" (HM, p. I07) sont justement les attributs définissant la musique : «L'objet intangible, inflairable, inattingible, invisible, asème, inexistant de la musique.» (HM, p. II2.) En cela, on touche une autre tension qui traverse la pensée de Quignard, qui concerne les conceptions sacrée et profane de la musique. Chez lui le sens du rite, du divin, de l'unique est extrêmement présent, comme on peut le voir dans le traité "Désenchanter». Reconduisant une nostalgie de l'expérience musicale telle qu'elle a pu se développer avant une certaine modernité, son désir de musique rejoint un désir de sacré :

Loccasion de la musique, pendant des millénaires, fut aussi singulière, intransportable, exceptionnelle, solennelle, ritualisée que pouvaient l'être une assemblée de masques, une grotte souterraine, un sanctuaire, un palais princier ou royal, des funérailles, un mariage. ( $H M$, p. 258.)

La musique multipliée à l'infini comme la peinture reproduite dans les livres, les magazines, les cartes postales, les films, les CD-ROM, se sont arrachées à leur unicité. Ayant été arrachées à leur unicité, elles ont été arrachées à leur réalité. Ce faisant, elles se sont dépouillées de leur vérité. Leur multiplication les a ôtées à leur apparition. Les ôtant à leur apparition, elle les a ôtées à la fascination originaire, à la beauté. (HM, p. 256-257.)

L'énumération qui, quelques pages plus loin, met sur le même plan «la parole, le chant, le poème et la prière» (HM, p. 263) va dans ce sens.

Pour autant, la genèse profane qu'il a proposée auparavant, dans le VIII ${ }^{e}$ traité, semble participer d'un tout autre horizon de référence :

I2. Sur le caractère divin de ces attributs, voir : "L'inconsistance et la non-délimitation sont des attributs divins.» (HM, p. II4.) 
Qu'est-ce que Dieu? Que nous soyons nés.

Que nous soyons nés d'autres que nous-mêmes. Que nous soyons nés dans un acte où nous ne figurions pas. Que nous soyons nés au cours d'une étreinte où deux autres corps que le nôtre étaient nus : que nous voulons voir.

Il se trouve que, remuant l'un vers l'autre, ils gémissent.

Nous sommes le fruit d'une secousse entre deux bassins dénudés, incomplets, honteux l'un devant l'autre, dont l'union était bruyante, rythmée, gémissante. (HM, p. 204-205.)

Accoler l'inconnaissable de la scène primordiale, la «nuit sexuelle» à la dimension intouchable du divin, autrement dit, profaner le sacré, peut-être est-ce un revirement qui rend au lieu du sacré une force subversive au sein de la modernité.

Ainsi, coupée ou non coupée de l'origine, défense quant au sonore originaire, souvenir ou consolation de la séparation, manifestation du sacré ou du profane, la musique, dans les deux textes, prend de multiples visages, aussi divers que contradictoires. On l'aura compris, plus qu'une tentative de définition de la musique, de ses attributs et des réactions qu'elle peut susciter, La Haine de la musique et Boutès relèvent bien plus de la mythologie personnelle. Du reste, à la lecture des dernières pages de Boutès, notamment quand l'auteur revient longuement sur son propre rapport à la musique, sa honte d'avoir laissé la musique "en souffrance» $(B$, p. 84$)$, de ne pas avoir honoré l'héritage familial, il est difficile de ne pas faire un rapprochement entre cette formule - la musique en souffrance - et la question qui ouvre La Haine de la musique: "J'interroge les liens entre la musique et la souffrance sonore." (HM, p. I6.) La souffrance sonore serait aussi (et avant tout) une souffrance propre à l'auteur lui-même. Quignard nous offre donc une méditation sur la musique qui ouvre des pistes de réflexion, dans une écriture poétique, dense, émouvante, mais qu'il faut très certainement aborder comme le déroulement d'obsessions singulières, dont la valeur de vérité est éminemment subjective, et signe une aimantation puissante.

\section{Haïr, aimer : d'une passion l'autre}

Malgré le déplacement d'un affect à l'autre dont procèdent ces deux textes, la persistance de la fascination semble indéniable : il s'y maintient un lien hautement passionnel qui sauve certes l'amour, mais qui laisse entières, au fond, les conditions de possibilités de la haine. En effet, sans doute pouvonsnous lire Boutès comme une des manières de "sauver la source", ce que Pascal Quignard évoque, dans La Haine de la musique, comme étant son délire : 
Sauver la source, tel est mon délire. Sauver la source du fleuve que la source engendre et que le fleuve engloutit à force de l'accroître. On fouille Troie et on pèle un oignon infini. Les grandes cités des temps anciens ne sont pas retournées à l'état des forêts qu'elles avaient défrichées. Elles n'y retourneront pas. Les civilisations laissent place dans le meilleur des cas à des ruines. Dans le pire, à des déserts irréversibles. Je fais partie de ce que j’ai perdu. (HM, p. I82.)

Faire partie de ce qu'on a perdu, ce n'est pas assumer pleinement la perte, c'est redonner à l'objet perdu une fonction de contenant - où l'objet n' est pas résolument détaché du sujet, mais, au contraire, l'englobe. Autrement dit, c'est transiger avec cette autre formule qui perce pourtant dans La Haine: «Dire, c'est perdre.» (HM, p. 24I.) Du reste, consacrer, avec Boutès, tout un livre à cette figure du plongeur, à celui qui revient à la source, avec les marques de positivité qui lui sont associées, c'est bel et bien maintenir la force de «la fascination originaire». ( $H M$, p. 257.)

De même, la valorisation du silence qui traverse Boutès demande à être mise en rapport avec les développements de La Haine à ce propos. Pour se convaincre de l'importance du silence dans Boutès, on notera simplement ce petit fait très significatif : tranchant par son aspect autobiographique, le dernier chapitre clôt le livre sur l'évocation du renoncement à la musique par Quignard lui-même, et, du point de vue textuel, sur la description d'une vision, qui rompt avec la poétique de résonance signifiante tenue jusqu'alors. Le mutisme de l'image s'associe une certaine écriture blanche :

Avant le service, une petite porte donnait sur les toilettes? Il y avait un tapis grisâtre. La lunette était en bois foncé verni. Sur le mur était encastré un minuscule lavabo à peine assez grand pour contenir mes mains, une savonnette qui sentait l'eau de Cologne, un gant de toilette, un miroir, un tube au néon. Une serviette pendait à un clou. $(B$, p. 89 .)

De là, il semble que le goût du silence développé dans Boutès est lui aussi en droite ligne de ce que manifeste La Haine sur la question. Dans le traité "Désenchanter", Quignard donne en effet au silence la qualité d'être "le vertige moderne" (HM, p. 254) autrement dit, la fonction d'être une résistance à l'audition massive et à la reproductibilité technique qui serait l'enfer musical d'aujourd'hui - ce en quoi la musique aurait passé la limite du bruit (HM, p. 250), de la «noise», du «vacarme» (HM, p. 268). Visant à subvertir ce qui est selon lui l'ordre dominant actuel de la pratique musicale, il prône le silence - ou, du moins, la rareté - posture assimilée ailleurs à l'anachorèse et à l'exil, eux aussi très valorisés : "J'aurais voulu relancer l'épidémie d'anachorèse des anciens Romains, lorsque Auguste imposa dans le sang l'empire, ou l'exil baroque des Solitaires [...]» (HM, p. 273). Ce faisant, néanmoins, il promeut justement la «fascination originaire» (HM, p. 257) : 
les attributs de la musique qui constituaient ailleurs les raisons de sa haine sont finalement maintenus, simplement nimbés de positivité : "C'est à la campagne qu'il m'arrive de rejouer avec plaisir, quelques instants, de cette chose ancienne, exceptionnelle, convocative, fascinante qui avait pour nom la "musique”.» (HM, p. 252.) À l'aliénation déplorée de l'envahissante musique d'aujourd'hui, à sa jouissance incessante, il répond simplement par la jouissance du mutisme - ou, du moins, par une jouissance rare et solitaire. À ce titre, ici encore, la dernière page du livre est importante : en évoquant le retrait de la marquise de Merteuil dans sa propriété de Jargeau, et en fermant le texte sur l'image de cette femme chantant «Joy» et "Ô solitude» de Purcell, il s'agit bien de proposer, comme alternative à la jouissance massive et collective, une jouissance solitaire.

Peut-être, finalement, les textes de Quignard ne sortent-ils pas vraiment de la haine - ou de ce qui lui ouvre ses portes, c'est-à-dire la fascination. Pour autant, à défaut de "solution" à la haine, ils ne proposent pas moins un ensemble de questions stimulantes, fondamentales, et là n'est pas la moindre de leurs qualités :

De quoi la musique est-elle l'instrument?

Quelle est l'intonation originaire de la musique? Pourquoi y a-t-il des instruments de musique?

Pourquoi les mythes sont-ils attentifs à leur naissance? (HM, p. II9.)

Pourquoi la naissance de l'art se trouva-t-elle liée à une expédition souterraine?

Pourquoi l'art fut-il et demeure-t-il une sombre aventure? (HM, p. I44-I45.)

Pourquoi la musique a-t-elle pu être «mêlée à l'exécution de millions d'êtres humains?» Pourquoi y prit-elle une "part plus qu'active»? (HM, p. 202.)

Comment entendre la musique, n’importe quelle musique, sans lui obéir?

Comment entendre la musique à partir du dehors de la musique? (HM, p. 208.)

Déroulant des réponses fluctuantes, réversibles, spécifiques à l'imaginaire quignardien, La Haine de la musique et Boutès poussent malgré tout le lecteur à penser, à élaborer ses propres hypothèses et ses associations.

S'il s'agissait néanmoins d'envisager une issue possible à la fascination de la musique, peut-être, simplement, faudrait-il se résoudre à l'évidence suivante : il faut faire de la musique! Car passer par la pratique musicale, cela permet de rompre la passivité de l'audition, les revers de la reproductibilité ainsi que la sacralité de l'événement. 
